\title{
Hybrid dynamic modeling of Escherichia coli central metabolic network combining Michaelis-Menten and approximate kinetic equations
}

\author{
Rafael S. Costa, Daniel Machado, Isabel Rocha, Eugénio C. Ferreira* \\ IBB-Institute for Biotechnology and Bioengineering, Centre of Biological Engineering, Universidade do Minho, Campus de Gualtar, 4710-057 Braga, Portugal
}

\section{A R T I C L E I N F O}

\section{Article history:}

Received 5 November 2009

Received in revised form 1 March 2010

Accepted 4 March 2010

\section{Keywords:}

Dynamic modeling

Escherichia coli metabolic network

Approximate rate equations

Parameter optimization

\begin{abstract}
A B S T R A C T
The construction of dynamic metabolic models at reaction network level requires the use of mechanistic enzymatic rate equations that comprise a large number of parameters. The lack of knowledge on these equations and the difficulty in the experimental identification of their associated parameters, represent nowadays the limiting factor in the construction of such models. In this study, we compare four alternative modeling approaches based on Michaelis-Menten kinetics for the bi-molecular reactions and different types of simplified rate equations for the remaining reactions (generalized mass action, convenience kinetics, lin-log and power-law). Using the mechanistic model for Escherichia coli central carbon metabolism as a benchmark, we investigate the alternative modeling approaches through comparative simulations analyses. The good dynamic behavior and the powerful predictive capabilities obtained using the hybrid model composed of Michaelis-Menten and the approximate lin-log kinetics indicate that this is a possible suitable approach to model complex large-scale networks where the exact rate laws are unknown.
\end{abstract}

(c) 2010 Elsevier Ireland Ltd. All rights reserved.

\section{Introduction}

One of the great challenges in the post-genomic era is to understand the dynamic behavior of a living cell. For that purpose, quantitative models describing metabolic network dynamics are a powerful tool to explain properties of complex biological systems and to guide experimentation (Kitano, 2002). In this context, dynamic changes in metabolite concentration over time are predominantly simulated using non-linear ordinary differential equations (Bakker et al., 1999; Klipp et al., 2007) that require a previous knowledge on the network structure and a large amount of experimental information, such as initial concentrations of metabolites, kinetic parameters and detailed kinetic rate laws. Mechanistic kinetic rate expressions have been the usual approach in metabolic networks modeling. In the last years, several dynamic models have been developed and examples include the tricarboxylic acid cycle in Dictyostelium discoideum (Wright et al., 1992), the threonine synthesis pathway in Escherichia coli (Chassagnole et al., 2001), and the glycolysis in Trypanosoma brucei (Bakker et al., 1997). A major challenge with such models, however, is that they often possess many kinetic parameters. While information

\footnotetext{
* Corresponding author. Tel.: +351253604 407; fax: +351 253678986.

E-mail addresses: rafacosta@deb.uminho.pt (R.S. Costa), dmachado@deb.uminho.pt (D. Machado),irocha@deb.uminho.pt (I. Rocha), ecferreira@deb.uminho.pt (E.C. Ferreira).
}

on network structure can be compiled from public databases (for example Karp et al., 2000; Ren and Paulsen, 2007), there are currently few methods for estimating kinetic parameters. In addition, kinetic parameter values measured in vitro may originate significant differences between simulated and experimental data because the conditions at which in vitro assays are performed are often different from those inside the cell (such as buffering conditions, temperature, intracellular $\mathrm{pH}$, etc.) (Richey et al., 1987; Teusink et al., 2000). A common approach to address this issue has been the use of time course in vivo data in response to a stimulus (Theobald et al., 1997; Vaseghi et al., 1999; Wahl et al., 2006) for kinetic parameter estimation by minimizing a cost function (Mendes and Kell, 1998; Moles et al., 2003). However, despite a number of successful applications, this approach has several limitations due to parameter identifiability problems on mechanistic models. On the other hand, the true mechanistic kinetic rate law for a specific reaction is frequently not known for most of the enzymes.

For these reasons, the application of these approaches to kinetic models requires a large amount of experimental data and has been limited to biochemical networks of limited size (Ishii et al., 2007), with the exception of the human red blood cell model (Jamshidi et al., 2001). Alternatively, large-scale metabolic models can be constructed based on stoichiometry without large fitted parameter sets. Although these models can be used to predict steady-state behavior using flux analysis, they fail to capture the transient behaviors of metabolism. Recently, a great effort has been car- 
ried out by researchers to develop alternative approaches to build large-scale models with incorporation of some dynamic phenomena. Dynamic flux balance analysis (DFBA) has been proposed for situations where there is kinetic knowledge available for part of the model (Mahadevan et al., 2002). Another hybrid approach was developed by Yugi et al. (2005). The proposed method aims to reduce the number of enzyme kinetic assays necessary to build a dynamic model, by considering a dynamic and a static part. The static module is simulated by metabolic flux analysis (MFA) constrained by the information obtained from the dynamic module. There are some limitations to be considered for accurate simulations, such as the need to know the elasticity coefficients at boundary reactions between modules and inconsistencies in the static module caused by the inclusion of irreversible reactions.

Another approach to overcome some of the difficulties in large-scale dynamic modeling is to use different alternative rate equations like linear-logarithmic (lin-log), logarithmic-linear (loglin), or power-law kinetics (Hatzimanikatis and Bailey, 1996; Savageau, 1970; Visser and Heijnen, 2002), where the kinetic parameter values can be estimated from time course and/or steadystate experimental data (Nikerel et al., 2006; Vilela et al., 2008). Parameters of such alternative kinetics expressions can be also inferred from the stoichiometry of the reactions (Smallbone et al., 2007). The most important advantage of approximate rate equations is the relative small number of parameters, which consequently reduces the number of experimental assays necessary for their identification, while still giving a reasonable description of the in vivo system. These approximated kinetic expressions also have a uniform format and therefore reduced mathematical complexity.

A number of dynamic simulation studies of biochemical systems with simplified enzyme kinetic equations have been reported in the literature. Spieth et al. (2006) applied linear weight matrices, $\mathrm{S}$-systems and $\mathrm{H}$-systems models, and also different optimization algorithms to model a non-linear dynamic system. Wang et al. (2007) compared S-Sytems and lin-log models to represent an aspartase-overproducing $E$. coli strain in batch fermentations. Furthermore, Voit et al. (2006) combined in vivo metabolite data obtained with NMR techniques and Biochemical Systems Theory as modeling framework to analyze the dynamic behavior in Lactococcus lactis. However, the applicability of multiple kinetic rate types for modeling large-scale biochemical networks has rarely been investigated.

In this work, we explore alternative approaches based on mechanistic (Michaelis-Menten) and simplified alternative kinetics to the large-scale E. coli network (Chassagnole et al., 2002). We constructed four hybrid models where the mechanisms for reactions with one substrate have been consider to follow the Michaelis-Menten kinetics, while approximated kinetics have been used for the other reactions, such as, generalized mass action, convenience kinetics, power-law, and lin-log. These models were then compared with the full mechanistic reference model (Chassagnole et al., 2002). Finally, analysis of stability and prediction power has been performed to evaluate the best alternative modeling approach.

\section{Methods}

Dynamic modeling of biochemical networks has evolved substantially in recent times, aided by the arrival of completely sequenced genomes (Blattner et al., 1997), the development of high-throughput technologies to rapidly obtain quantitative measurements for multiple metabolites (Theobald et al., 1993; Visser et al., 2002), and the completion of publicly-available metabolic databases (Ji et al., 2003; Karp et al., 2000; Schomburg et al., 2002; Sundararaj et al., 2004; Rojas et al., 2007). Non-linear ordinary differential equations (ODEs) systems are the most commonly applied technique to model quantitatively a biochemical network. If we consider $n$ species, the generic form will come (Conrad and Tyson, 2006):

$\frac{\mathrm{d} C_{i}}{\mathrm{~d} t}=\sum_{j=1}^{m} N_{i j} r_{j}-\mu C_{i}$

where $C_{i}$ is the concentration of metabolite $i, N_{i j}$ is the stoichiometric coefficient of metabolite $i$ in reaction $j$. The reaction rate $r_{j}$ of the $j$ th reaction is given by non-linear expressions, which depend on the metabolite concentrations and kinetic parameters, $\mu$ is the specific growth rate and $m$ is the number of reactions in the network.

\subsection{Reference Model}

In this contribution, the full mechanistic model representing the central carbon metabolism of $E$. coli formulated by Chassagnole et al. (2002), available in SBML curated format at BioModels online database (Le Novere et al., 2006), was selected as a benchmark. The model integrates the reactions of glycolysis, pentose phosphate pathway, the phosphotransferase system (PTS), and several compoundsynthesis systems connected with these pathways that lead for biomass formation. The details of the metabolic network, the mass balances, the initial concentrations of the metabolites, and the list of mathematical mechanistic rate equations of the model can be found in the original paper (Chassagnole et al., 2002). The model accounts for 30 reaction rates, outlined in Table 1, with a total of 116 kinetic parameters and 18 metabolites. The co-metabolites were represented by time-dependent non-linear functions (Chassagnole et al., 2002). The model simulations using the deterministic LSODA solver (Petzold, 1983) were conducted during $40 \mathrm{~s}$ after a glucose impulse of $1.67 \mathrm{mM}$.

\subsection{Kinetic Rate Equations for Dynamic Analysis}

\subsubsection{Michaelis-Menten}

The Michaelis-Menten kinetics assumes that the rate at which an enzyme binds to its substrate is much faster than the rate of the product formation, and that the intermediate reaction is therefore at steady-state (see for example Lauffenburger and Linderman, 1993). The reaction rate is dependent on the rate constants Michaelis constant, $K_{\mathrm{M}}$, and forward rate constant, $r_{\max }$. The Michaelis-Menten rate expression can be applied to reactions with one substrate and one product (bimolecular reactions), and the more complex expression considering inhibition and reversibility (Cornish-Bowden, 1995; Heinrich and Schuster, 1996) can be written as:

$r=\frac{\left(r_{\max }^{+} / K_{\mathrm{M}}^{\mathrm{S}}\right) S-\left(r_{\max }^{-} / K_{\mathrm{M}}^{\mathrm{P}}\right) P}{1+I K_{\mathrm{I}}^{a}+\left(\left(S / K_{\mathrm{M}}^{\mathrm{S}}\right)+\left(P / K_{\mathrm{M}}^{\mathrm{P}}\right)\right)\left(1+I K_{\mathrm{I}}^{b}\right)}$

where $K_{\mathrm{M}}^{\mathrm{S}}$ and $K_{M}^{\mathrm{P}}$ are the Michaelis-Menten constants for the substrate and product, respectively. $K_{\mathrm{I}}^{a}$ and $K_{\mathrm{I}}^{b}$ are the inhibition constants. $r_{\max }^{+}$is the maximal forward reaction rate and $r_{\max }^{-}$the maximal reverse reaction rate. $S$, $P$ and $I$ denote the concentrations of the substrate, product, and inhibitor, respectively. To describe the three inhibition effects the following limits are often used (Heinrich and Schuster, 1996): for uncompetitive inhibition $\left(K_{\mathrm{I}}^{a} \rightarrow+\infty\right.$ and $\left.0<K_{\mathrm{I}}^{b}<+\infty\right)$, competitive inhibition $\left(K_{\mathrm{I}}^{b} \rightarrow+\infty\right.$ and $\left.0<K_{\mathrm{I}}^{a}<+\infty\right)$ and non-competitive inhibition $\left(0<K_{\mathrm{I}}^{a}=K_{\mathrm{I}}^{b}<+\infty\right)$.

If the mechanism of the reaction is not affected by an inhibitor and the thermodynamic equilibrium constant $\left(K_{\mathrm{eq}}=r_{\max }^{+} K_{\mathrm{M}}^{\mathrm{P}} / r_{\max }^{-} K_{\mathrm{M}}^{\mathrm{S}}\right)$ is known, then the rate law can be reduced to the following equation:

$r=\frac{r_{\max }\left(S-\left(P / K_{\mathrm{eq}}\right)\right)}{K_{\mathrm{M}}^{\mathrm{S}}\left(1+\left(P / K_{\mathrm{M}}^{\mathrm{P}}\right)\right)+S}$

\subsubsection{Approximate Kinetics Representation}

In general, the precise enzyme kinetic rate laws are not known for all the enzymes and membrane transporters. The approximated kinetic representations that we propose are the generalized mass action (Horn and Jackson, 1972; Schauer and Heinrich, 1979), lin-log kinetics (Hatzimanikatis and Bailey, 1997; Visser and Heijnen, 2003), power-law models (Savageau and Voit, 1982), and the more recently developed convenience rate law (Liebermeister and Klipp, 2006).

2.2.2.1. Generalized mass action. The generalized mass action (GMA) represents the simplest rate law, in which the enzymes effects are hidden. This rate law requires the specification of only two rate constants, the forward rate constant $(k)$ and the thermodynamic equilibrium constant $\left(K_{\mathrm{eq}}\right)$. The value of the constant $k$ takes into account all unknown effects influencing the enzyme, like the allosteric effectors or molecular crowding. If inhibition and/or activation are involved, a positive pre-function has to be applied, as suggested by Schauer and Heinrich (1983) and Liebermeister and Klipp (2006). Therefore, the rate equation takes the following form:

$r=k \prod_{l^{\prime}=1}^{n A}\left(\frac{A_{l^{\prime}}}{A_{l^{\prime}}+K_{\mathrm{A}}^{l^{\prime}}}\right)^{W_{l^{\prime}}} \prod_{l=1}^{n I}\left(\frac{1}{1+K_{\mathrm{I}}^{l} I_{l}}\right)^{W_{l^{-}}}\left(\prod_{i=1}^{n S} S_{i}{ }^{g_{i}}-\frac{1}{K_{\mathrm{eq}}} \prod_{j=1}^{n P} P_{j}{ }^{h_{j}}\right)$ 
Table 1

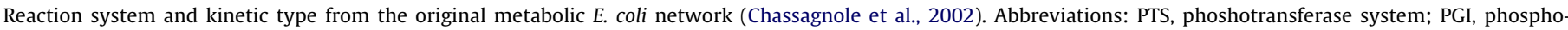

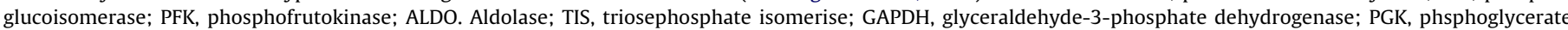

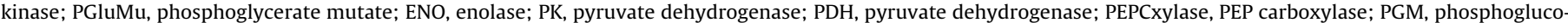

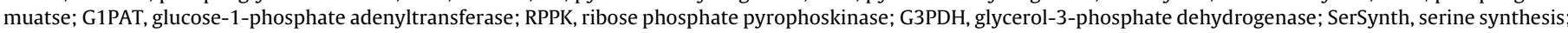

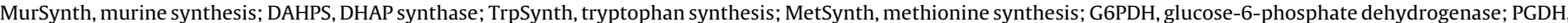

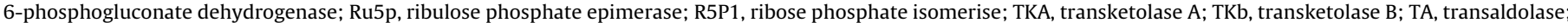
Synth 1 , synthesis 1 ; Synth 2, synthesis 2 .

\begin{tabular}{|c|c|c|c|c|}
\hline Reaction (EC number) & Reaction mechanism $^{a}$ & Inhibitors & Activators & Kinetic mechanism (no. of parameters) \\
\hline PTS $(-)$ & $\mathrm{PEP}+\mathrm{GLCEX} \rightarrow \mathrm{G} 6 \mathrm{P}+\mathrm{PYR}$ & G6P & & PTS rate equation from ${ }^{\mathrm{b}}(6)$ \\
\hline PGI (5.3.1.9) & $\mathrm{G} 6 \mathrm{P} \leftrightarrow \mathrm{F} 6 \mathrm{P}$ & $6 P G$ & & Reversible MM (6) \\
\hline PFK (2.7.1.11) & $\mathrm{F} 6 \mathrm{P}+\mathrm{ATP} \rightarrow \mathrm{FDP}+\mathrm{ADP}$ & PEP & AMP, ADP & Four state allosteric model (11) \\
\hline ALDO (4.1.2.13) & $\mathrm{FDP} \leftrightarrow \mathrm{GAP}+\mathrm{DHAP}$ & & & Ordered uni-bi reaction (7) \\
\hline TIS (5.3.1.1) & $\mathrm{DHAP} \leftrightarrow \mathrm{GAP}$ & & & Reversible MM (4) \\
\hline GAPDH (1.2.1.12) & $\mathrm{GAP}+\mathrm{NAD} \leftrightarrow \mathrm{PGP}+\mathrm{NADH}$ & & & Two substrate reversible MM (6) \\
\hline PGK (2.7.2.3) & $\mathrm{PGP}+\mathrm{ADP} \leftrightarrow 3 \mathrm{PG}+\mathrm{ATP}$ & & & Two substrate reversible MM (6) \\
\hline PGluMu (5.4.2.1) & $3 \mathrm{PG} \leftrightarrow 2 \mathrm{PG}$ & & & Reversible MM (4) \\
\hline ENO (4.2.1.11) & $2 \mathrm{PG} \leftrightarrow \mathrm{PEP}+\mathrm{H}_{2} \mathrm{O}$ & & & Reversible MM (4) \\
\hline PK (2.7.1.40) & $\mathrm{PEP}+\mathrm{ADP} \rightarrow \mathrm{PYR}+\mathrm{ATP}$ & ATP & FDP, AMP & Allosteric regulation based on ${ }^{\mathrm{c}}(8)$ \\
\hline $\mathrm{PDH}(1.2 .4 .1)$ & $\mathrm{PYR}+\mathrm{NAD}^{+}+\mathrm{COA} \rightarrow \varnothing$ & & & Hill equation ( 3 ) \\
\hline PEPCxylase (4.1.1.31) & $\mathrm{PEP}+\mathrm{H}_{2} \mathrm{O}+\mathrm{CO}_{2} \rightarrow \varnothing$ & & FDP & Empirical equation based on ${ }^{\mathrm{d}}(4)$ \\
\hline PGM (5.4.2.2) & $\mathrm{G} 6 \mathrm{P} \leftrightarrow \mathrm{G} 1 \mathrm{P}$ & & & Reversible MM (4) \\
\hline G1PAT (2.7.7.27) & $\mathrm{G} 1 \mathrm{P}+\mathrm{ATP} \rightarrow \varnothing$ & & FDP & Empirical two substrate eq. ${ }^{\mathrm{e}}(5)$ \\
\hline RPPK (2.7.6.1) & $\mathrm{RIB5P}+\mathrm{ATP} \rightarrow \varnothing$ & & & Irreversible MM (2) \\
\hline G3PDH (1.1.1.94) & $\mathrm{DHAP}+\mathrm{NADPH} \rightarrow \varnothing$ & & & Irreversible MM (2) \\
\hline SerSynth $(-)$ & PG3 $\rightarrow \varnothing$ & & & Irreversible MM (2) \\
\hline MurSynth (-) & $2 \mathrm{~F} 6 \mathrm{P} \rightarrow \varnothing$ & & & Constant level (steady-state flux) (1) \\
\hline DAHPS (2.5.1.54) & $\mathrm{PEP}+\mathrm{E} 4 \mathrm{P}+\mathrm{H}_{2} \mathrm{O} \rightarrow \varnothing$ & & & Two substrate Hill equation (5) \\
\hline TrpSynth (-) & $\varnothing \rightarrow$ PYR + GAP & & & Constant level (steady-state flux) (1) \\
\hline MetSynth (-) & $\varnothing \rightarrow$ PYR & & & Constant level (steady-state flux) (1) \\
\hline G6PDH (1.1.1.49) & $\mathrm{G} 6 \mathrm{P}+\mathrm{NADP} \rightarrow 6 \mathrm{PG}+\mathrm{NADPH}$ & NADPH & & Two substrate equation based on ${ }^{f}$ without ATP inhibition (5) \\
\hline PGDH (1.1.1.44) & $6 \mathrm{PG}+\mathrm{NADP} \rightarrow \mathrm{RIBU} 5 \mathrm{P}+\mathrm{CO}_{2}+\mathrm{NADPH}$ & NADPH, ATP & & Two substrate eq. based on ${ }^{\mathrm{f}}(5)$ \\
\hline Ru5p (5.1.3.1) & RIBU5P $\leftrightarrow \mathrm{XYL5P}$ & & & Reversible mass action (2) \\
\hline R5P1 (5.3.1.6) & RIBU5P $\leftrightarrow$ RIB5P & & & Reversible mass action (2) \\
\hline TKa (2.2.1.1) & $\mathrm{XYL5P}+\mathrm{RIB5P} \leftrightarrow \mathrm{GAP}+\mathrm{SED7P}$ & & & Reversible mass action (2) \\
\hline $\mathrm{TKb}(2.2 .1 .1)$ & $\mathrm{E} 4 \mathrm{P}+\mathrm{XYL5P} \leftrightarrow \mathrm{F} 6 \mathrm{P}+\mathrm{GAP}$ & & & Reversible mass action (2) \\
\hline $\mathrm{TA}(2.2 .1 .2)$ & $\mathrm{SED7P}+\mathrm{GAP} \leftrightarrow \mathrm{F} 6 \mathrm{P}+\mathrm{E} 4 \mathrm{P}$ & & & Reversible mass action (2) \\
\hline Synth $1(-)$ & $\mathrm{PEP} \rightarrow \varnothing$ & & & Irreversible MM (2) \\
\hline Synth $2(-)$ & $\mathrm{PYR} \rightarrow \varnothing$ & & & Irreversible MM (2) \\
\hline
\end{tabular}

MM denotes Michaelis-Menten kinetic and the symbol $\varnothing$ represent contribution for biomass synthesis.

a Chassagnole et al. (2002).

b Liao et al. (1996).

c Johannes and Hess (1973).

d Kameshita et al. (1979).

e Preiss et al. (1975).

f Vaseghi et al. (1999).

where the positive integer constants $W_{l^{\prime}}^{+}$and $W_{l^{\prime}}^{-}$are defined as the connectivity of the activator (A) or inhibitor (I) metabolites. The constants $g_{i}$ and $h_{j}$ are the stoichiometric coefficients with which the $i$ th substrate and $j$ th product enter the reaction. $K_{\mathrm{A}}^{l^{\prime}}$ and $K_{\mathrm{I}}^{l}$ denote the activation and inhibition constants for the $l$ th inhibitor and $l$ th activator, respectively. $n A, n I, n S$ and $n P$ are respectively the total number of activators, inhibitors, substrates, and products.

2.2.2.2. Lin-Log. The non-mechanistic lin-log representation (Visser and Heijnen, 2003 ) is based on the notion that the rate of reaction and the thermodynamic driving force are proportional. The rate laws of all reactions have the same mathematical structure with linearity in the parameters called elasticities $\left(\varepsilon_{\mathrm{S}}^{0}\right.$ and $\left.\varepsilon_{\mathrm{p}}^{0}\right)$ and the effect of metabolite levels on the reaction rates being described as a sum of logarithmic concentration terms, represented by:

$r=r^{0} \frac{e}{e^{0}}\left(1+\sum_{i=1}^{n S} \varepsilon_{S i}^{0} \ln \left(\frac{S_{i}}{S_{i}^{0}}\right)+\sum_{j=1}^{n P} \varepsilon_{P j}^{0} \ln \left(\frac{P_{j}}{P_{j}^{0}}\right)\right)$

where the superscripts $\left({ }^{0}\right)$ denote the reference state (e.g. wild type at steady-state), $r^{0}$ is the reference reaction rate value, $e / e^{0}$ represent the relative enzyme activities, $S_{i} / S_{i}^{0}$ and $P_{j} / P_{j}^{0}$ are the relative concentrations that influence the kinetics of the reaction. The elasticities are defined as the scaled local partial derivatives of the $j$ th reaction rate with the metabolite $i$ :

$\varepsilon_{i, j}=\frac{C_{i}^{0}}{r_{j}^{0}} \frac{\delta r_{j}}{\delta C_{i}}$
The inclusion of inhibition and activation effects and transformation of Eq. (5) to facilitate parameter fitting originates:

$$
\begin{aligned}
r= & \frac{e}{e^{0}}\left(r^{0}+\sum_{i=1}^{n S} a_{i} \ln \left(\frac{S_{i}}{S_{i}^{0}}\right)+\sum_{j=1}^{n P} b_{j} \ln \left(\frac{P_{j}}{P_{j}^{0}}\right)+\prod_{l=1}^{n l} c_{l} \ln \left(\frac{I_{l}}{I_{l}^{0}}\right)\right. \\
& \left.+\prod_{l^{\prime}=1}^{n A} d_{l^{\prime}} \ln \left(\frac{A_{l^{\prime}}}{A_{l^{\prime}}^{0}}\right)\right)
\end{aligned}
$$

with the empirical constants $a_{i}$ for the substrates and $b_{j}$ for the products being now the semi-scaled elasticities at the reference state. The empirical constants $c_{l}$ and $d_{l}$ are for the inhibitors and activators, respectively. In our study, the $e / e^{0}$ is set to 1 assuming that the enzyme level remains constant during the simulation. The initial concentrations of the metabolites of the full mechanistic model were taken as our reference state.

2.2.2.3. Power-Law. The power-law representation was originally proposed by Savageau (1969). In this modeling framework, each reaction rate is represented as products of power-law functions that include all variables that affect the process The generic rate equation structure is given by:

$r=r^{0} \prod_{i=1}^{n S}\left(\frac{S_{i}}{S_{i}^{0}}\right)^{v_{i}} \prod_{j=1}^{n P}\left(\frac{P_{j}}{P_{j}^{0}}\right)^{w_{j}}$

where $v_{i}$ and $w_{j}$ are non-dimensional constants and $r^{0}$ is the reaction rate at the reference state. Also, $S^{0}$ and $P^{0}$ are the concentrations of substrates and products at a reference state. 
Table 2

Summary of the total number of parameters in the approximated models generated in this study and the original model.

\begin{tabular}{|c|c|c|c|c|c|}
\hline & \multicolumn{5}{|c|}{ Model name } \\
\hline & Original & GMA & Convenience & Lin-log & Power-law \\
\hline Total number of parameters & 116 & 77 & 115 & 96 & 110 \\
\hline
\end{tabular}

2.2.2.4. Convenience kinetics. The most recently developed semi-mechanistic rate law was formulated from a simple random-order enzyme mechanism and can be applied to any reaction catalyzed by an enzyme. This rate law is a particular case of the generalized mass action kinetics (for details on the equation derivation see Liebermeister and Klipp, 2006). The general representation of this equation is described by:

$$
\begin{aligned}
r=E \prod_{l^{\prime}=1}^{n A}\left(\frac{A_{l^{\prime}}}{A_{l^{\prime}}+K_{\mathrm{A}}^{l^{\prime}}}\right)^{W_{l^{+}}} \prod_{l=1}^{n I}\left(\frac{1}{1+K_{\mathrm{I}}^{l} I_{l}}\right)^{W_{l^{-}}} \\
\times \frac{k_{\mathrm{cat}}^{+} \prod_{i=1}^{n S}\left(\frac{S_{i}}{K_{\mathrm{M}, \mathrm{S}}^{i}}\right)^{\alpha_{i}}-k_{\mathrm{cat}}^{-} \prod_{j=1}^{n P}\left(\frac{P_{j}}{K_{\mathrm{M}, \mathrm{P}}^{j}}\right)^{\beta_{j}}}{\prod_{i=1}^{n S}\left(\sum_{m=0}^{\alpha_{i}}\left(\frac{s_{i}}{K_{\mathrm{M}, \mathrm{S}}^{i}}\right)^{m}\right)+\prod_{j=1}^{n P}\left(\sum_{m=0}^{\beta_{j}}\left(\frac{P_{j}}{K_{\mathrm{M}, \mathrm{P}}^{j}}\right)^{m}\right)-1}
\end{aligned}
$$

where $k_{\mathrm{cat}}^{+}$and $k_{\mathrm{cat}}^{-}$are the turnover constants, $K_{\mathrm{M}, \mathrm{S}}^{i}$ and $K_{\mathrm{M}, \mathrm{P}}^{i}$ are constants analogous to the Michaelis constant for the substrates and products, respectively; $\alpha$ and $\beta$ are the stoichiometric coefficients. Assuming that the enzyme concentration $E$ remains constant during the simulation time, the products $E k_{\text {cat }}^{+}$and $E k_{\text {cat }}^{-}$are replaced by $r_{\max }^{+}$and $r_{\max }^{-}$. Evidently, this kinetics requires comparatively more parameters than other approximated approaches.

\subsection{Parameter Estimation and Stability Analysis}

Given a set of experimental data and a model structure, the aim of parameter estimation is to calibrate the model by solving an optimization problem where the objective function represents the distance between the model and experimental data. The calibration of the models to the noise-free pseudo-experimental time series data sets generated by simulation using the full mechanistic $E$. col model (reference model) after glucose impulse was performed using the Complex Pathway Simulator (Copasi) software tool v. 4.4 (Hoops et al., 2006). The metabolite concentration time series data sets were obtained at sampling interval of $0.5 \mathrm{~s}$. Parameter estimation was performed by using the evolutionary programming (EP) and the Hooke and Jeeves methods (Fogel et al., 1966; Hooke and Jeeves, 1961). We have considered the EP method for parameter estimation because evolutionary algorithms have proven to have key advantages in large inverse problems of quantitative mathematical models (Mendes, 2001). The goodness of fit for each set of estimated parameter values was quantified by the following fitness function:

$f_{\mathrm{val}}(p)=\sum_{i=1}^{s} \sum_{j=1}^{t} \omega_{i}\left(x_{i, j}-y_{i, j}(p)\right)^{2}$

where $y_{i, j}(p)$ and $x_{i, j}$ are time course points obtained using each of the four alternative models and the reference model, respectively. $p$ is the tested parameter set and $s$ and $t$ are the number of metabolites and data points, respectively. In addition, $\omega_{i}$ correspond to the different weights used to normalize the contributions of each term. In this study, we use the mean square, $\omega_{i}=1 / \sqrt{\left\langle x_{i}^{2}\right\rangle}$, to assure that columns with small values contribute in the same order of magnitude to Eq. (10). The estimation of kinetic parameters was performed separately for each of the four models formed by 18 ODEs and 30 reactions. As routine, the EP method was used to obtain an initial set of parameters and this solution was then refined using the local optimization method Hooke and Jeeves to yield the refined estimates.

The stability of the models was evaluated by calculating the eigenvalues of the Jacobian matrix for all parameters computed by Copasi. The kinetic model is stable if all the real parts of the eigenvalues are negative. The stiffness of the models was evaluated by calculating the ratio of the largest over the smallest eigenvalue, which evaluates the time step sizes needed to achieve a stable solution.

\subsection{Model Ranking and Selection}

The mean relative error (MRE) was used to evaluate the performance of each alternative modeling approach, defined as (Kitayama et al., 2006):

$\operatorname{MRE}(\%)=\left(\frac{\sum_{i=1}^{s} \sum_{i=1}^{t}\left|x_{i, j}-y_{i, j} / x_{i, j}\right|}{s t}\right) 100$

where $x_{i, j}$ is the pseudo-experimental data of a given $(i)$ metabolite concentration and $y_{i, j}$ is the concentration given by the model at the $j$ th sampling point $(t)$ and $s$ is the number of metabolites. Each distance is normalized to overcome the different orders of magnitude of the metabolite concentrations.

\subsection{Parameter Sensitivity}

The sensitivity of the kinetic parameters with respect to the reaction rates was calculated using the SBML-SAT software (Zi et al., 2008). A time-dependent normalized sensitivity response is defined by the following equation:

$S_{i j}(r(t), p)=\frac{\partial r_{j}(t) p_{i}}{\partial p_{i} r_{j}(t)}$

where $r_{j}(t)$ is the $j$ th rate law and $p_{i}$ is the $i$ th parameter.

\section{Results and Discussion}

For large-scale kinetic models we need extensive knowledge on the stoichiometry of the metabolic network, the kinetic parameters and detailed rate laws. An integration of these three types of information will in principle allow to describe the rate of change for each metabolite. However, it is often very difficult to determine the functional form of the rate equations for the majority of the biochemical reactions and their associated kinetic parameters. A general principle when building kinetic models is to make the model as simple as possible, while capturing the realistic dynamic behavior (Dano et al., 2006). As such, some alternative strategies have been proposed (Famili et al., 2005; Yugi et al., 2005). The motivation for this work is to evaluate alternative kinetic modeling approaches containing fewer kinetic parameters in a large-scale network. For this purpose, we compare four hybrid models combining Michaelis-Menten for bi-molecular enzyme reactions rate laws and approximate rate equations with the full mechanistic reference model for the central carbon metabolism of $E$. coli proposed in Chassagnole et al. (2002). By developing these kinetic models it was possible to simulate the entire $E$. coli network.

\subsection{Comparison of the Modeling Approaches}

The reactions, number of parameters, and the type of kinetic mechanism for each biochemical reaction in the reference model are summarized in Table 1. Due to the approximation assumptions of the alternative rate equations we have applied Michaelis-Menten equations whenever this was possible, in other words, to reactions with only one substrate. Moreover, the multimolecular reactions (with more than one substrate or product) assumed to exhibit Michaelis-Menten kinetics in the reference model (RPPK and G3PDH) are left unchanged in the alternative models. All other multimolecular reactions are replaced with simplified kinetics. The reactions MurSynth, TrpSynth, and MetSynth were considered at steady-state (Chassagnole et al., 2002) and therefore the corresponding kinetic laws were not replaced in the alternative models. Among all the alternative models, the one 

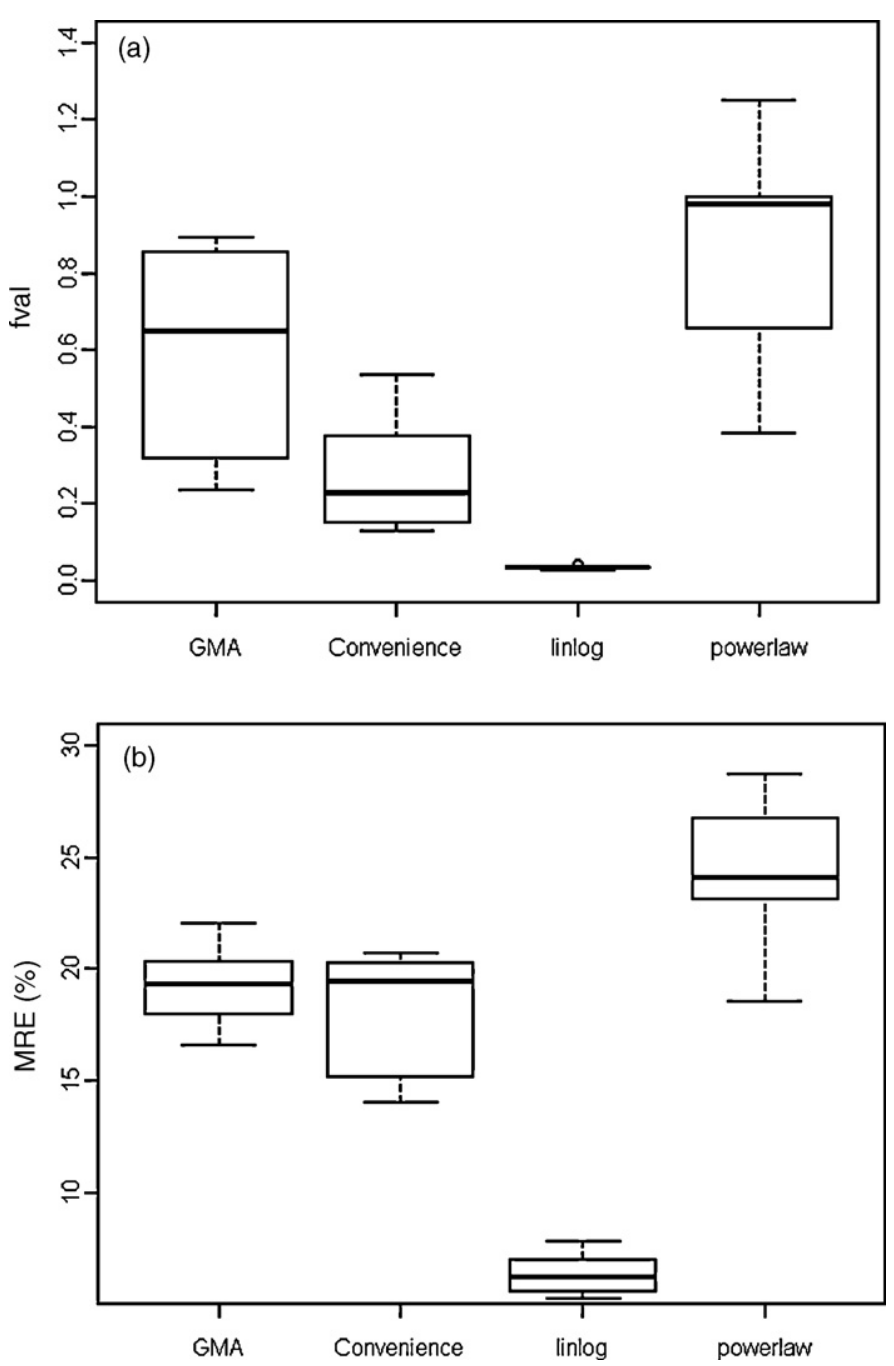

Fig. 1. Objective functions - (a) $f_{\text {val }}$ and (b) mean relative error (MRE) - obtained during parameter identification for each alternative model from 10 independent estimation runs for each case.

where multimolecular reactions have been described by the convenience kinetics contains the highest number of kinetic parameters (115 as compared with 116 of the original model). This is followed by the model based on power-law rate laws that contains 110 parameters, the lin-log system with 96 and the GMA model with only 77 kinetic parameters (Table 2 ).

Parameter estimation can be performed through local and/or global methods (Mendes and Kell, 1998; Rodriguez-Fernandez et al., 2006). However, one of the major challenges in modeling largescale dynamic systems is the existence of several local minima in the space of solutions. In this paper, we used an evolutionary programming method and its solution is then used as the starting point for a local search method ("Hooke and Jeeves"). To make sure that this method does not converge to a sub-optimal local minimum, 10 optimizations runs were performed with different random initial guesses.

After parameter estimation, we checked whether the alternative models are able to reproduce the same pseudo-experimental data used for parameter estimation. There exist a number of criteria to select among alternative candidate models. Here the $f_{\text {val }}$ (Eq. (10)) and MRE (Eq. (11)) methods were used as measures of the quality of the fit for an estimated parameter set, which allows comparisons among different data sets and models. The results are summarized in Fig. 1 . The mean and standard deviation of $f_{\text {val }}$ were calculated and among the four models, the lin-log $\left(f_{\text {val }}=0.0341 \pm 0.00357\right)$ achieved the best fits to the data followed by the convenience model $\left(f_{\text {val }}=0.272 \pm 0.138\right)$, the GMA model $\left(f_{\text {val }}=0.591 \pm 0.288\right)$, and the power-law model $\left(f_{\text {val }}=0.862 \pm 0.242\right)$. Similar conclusions can be drawn from analysis of MRE (Fig. 1b).

To obtain time series data, the reference steady-state model was perturbed by increasing the extracellular glucose concentration at time zero from $0.0556 \mathrm{mM}$ to $1.67 \mathrm{mM}$. The simulated time course data of the best fit solution for extracellular glucose, glyceraldehhyde-3-phosphate, glucose-1-phosphate, fructose-1,6bisphosphate, phosphoenolpyruvate, and pyruvate concentrations are shown in Fig. 2. In this figure we can observe that the discrepancies between the reference and alternative models were relatively small for most of the models in response to glucose addition, confirming also that the lin-log model gives the most accurate results and that it can successfully replace the full mechanistic model to represent the time course data along the $40 \mathrm{~s}$. For all the remaining metabolites, there is also a good agreement between the reference and the lin-log model (data not shown).

The average deviation (MRE) of the lin-log and convenience models to the reference simulated data for all metabolites was $6.37 \pm 0.87 \%$ and $18.21 \pm 2.71 \%$, respectively. For the GMA it was $19.36 \pm 1.83 \%$ and for power-law $24.42 \pm 3.30 \%$. The highest performance of the lin-log model for approximating enzyme kinetics is in line with a previous work from Heijnen (2005) for the single reaction level. In this work the authors describe the validity of lin-log kinetics for a single reaction and a higher performance was obtained when compared with other enzyme kinetics, such as the power-law formalism. Previous in silico and experimental studies have also shown a satisfactory performance of this nonlinear kinetics upon large changes in metabolites concentrations and fluxes (Visser and Heijnen, 2003). More importantly, the linlog model represents an option to simulate the dynamic behavior of the metabolic network with a lower number of kinetic parameters (96) compared with most of the other alternatives. Thus, the set-up of large-scale kinetic models based on this simplified model seems a promising approach to overcome the above-mentioned limitations.

A known limitation of the lin-log kinetics is that, for very small metabolite concentrations, it runs towards negative and the reaction rate is undefined. However, considering the homeostatic condition (i.e. steady-state) in the interior of the cell, this condition is unlikely to occur for metabolites in the $E$. coli central carbon metabolism. Another possible difficulty is the low sampling intervals of the metabolites required for parameter identification, but this is also a challenge of all the other kinetic models. For the parameter estimation, we assumed also that all the 18 metabolites are measurable. Even though this might be relevant at the present moment, the recent developments in high-throughput metabolomic methods that can be applied for such measurements will hopefully allow overcoming this issue (Buchholz et al., 2001; Soga et al., 2003). Finally, since lin-log models are obtained by a local approximation to a steady-state, it is likely that the quality of the results achieved in simulations deteriorates as we move away from this reference state. Taken these results together, the best performance of the lin-log model from a set of plausible candidates implied its selection for further analysis in this paper.

\subsection{Performance of the Lin-Log Model}

A requirement for a useful dynamic model is its stability. This can be achieved if all the eigenvalues of the Jacobian matrix for all parameter sets have negative real parts (Murray, 2002). To compare the stability of the lin-log model and the reference mechanistic model, we first computed the Jacobian matrix to determine all the eigenvalues for each complete ODEs system. If the real parts of the 

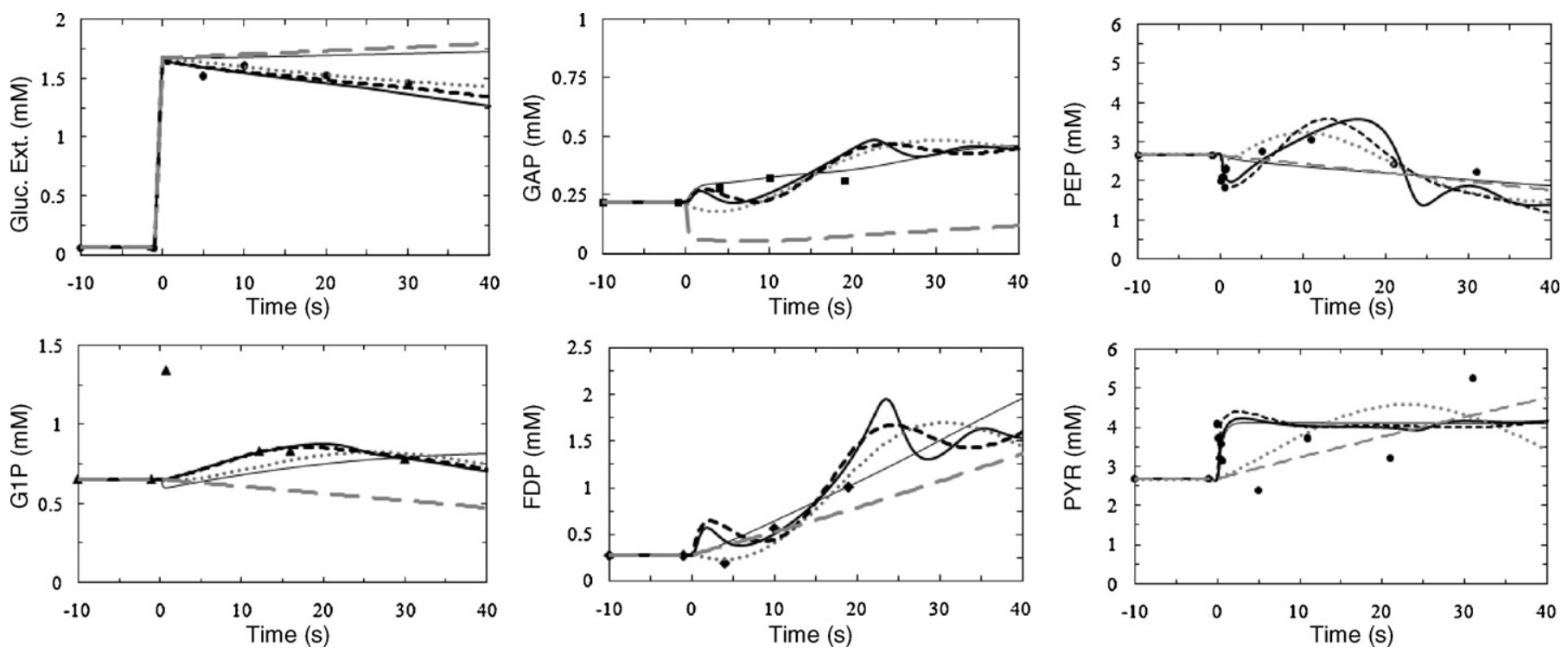

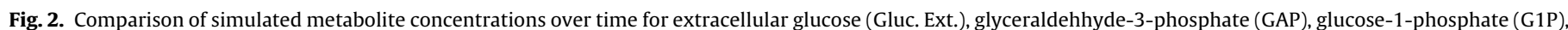

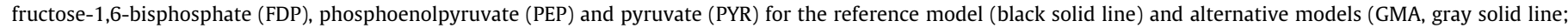

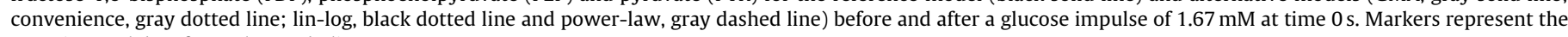
experimental data for each metabolite.

eigenvalues of both systems are negative than these models are stable and are able to return to the equilibrium after a perturbation in metabolite concentrations. The largest eigenvalue observed was $-0.0629 \mathrm{~s}^{-1}$ for the lin-log model and $-0.00853 \mathrm{~s}^{-1}$ for the reference model. Moreover, two complex eigenvalues were found for each model, indicating that the systems are able to oscillate, which corresponds to what can happen in real systems, as it has been shown experimentally (Buchholz et al., 2002).

In addition, the difference found in model stiffness $\left(4.0 \times 10^{5}\right.$ for the reference model and $4.4 \times 10^{5}$ for the lin-log model) means that for the lin-log model the numerical methods used for simulation require slightly smaller time steps to obtain stable solutions of the system.

To validate the lin-log model, experiments with data sets different from the ones used for parameter estimation were performed. Predictions under new conditions were made with the best param- eter set fixed. Thus, after a glucose impulse of $3.5 \mathrm{mM}$ and $10 \mathrm{mM}$, the MRE index was calculated and values of $10.19 \%$ and $15.96 \%$ were obtained, respectively. The time series results for some metabolites from the glycolysis and pentose phosphate pathways after a glucose impulse of $3.5 \mathrm{mM}$ are shown in Fig. 3. The behavior results indicate that the lin-log model, when compared with the reference model, has very good prediction levels during the $40 \mathrm{~s}$ time course for all the metabolites.

\subsection{Sensitivity Analysis of the Model}

Sensitivity analysis is also a tool for model validation and a measure of biological adequacy. This analysis quantified the effect that an infinitesimal change in the value of a parameter has on the steady-state values of the model, using usually methods of metabolic control analysis. In this study, however, we have focused
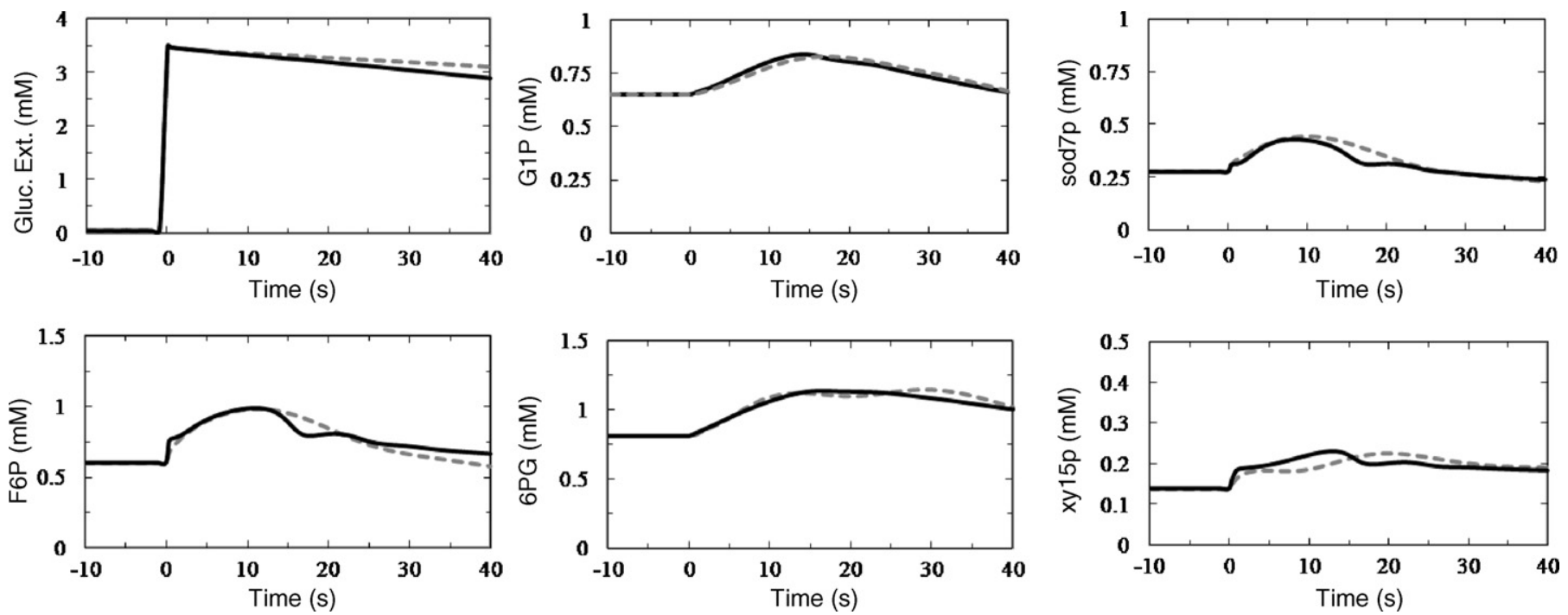

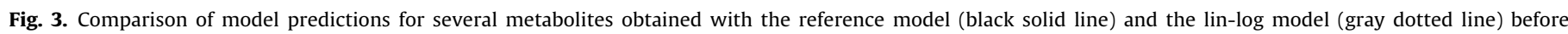

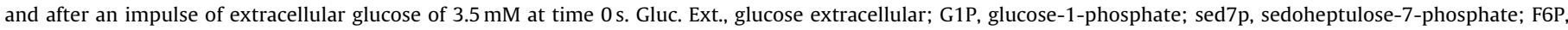
fructose-6-phosphate; 6PG, 6-phosphogluconate; xyl5p, xlulose-5-phosphate. 

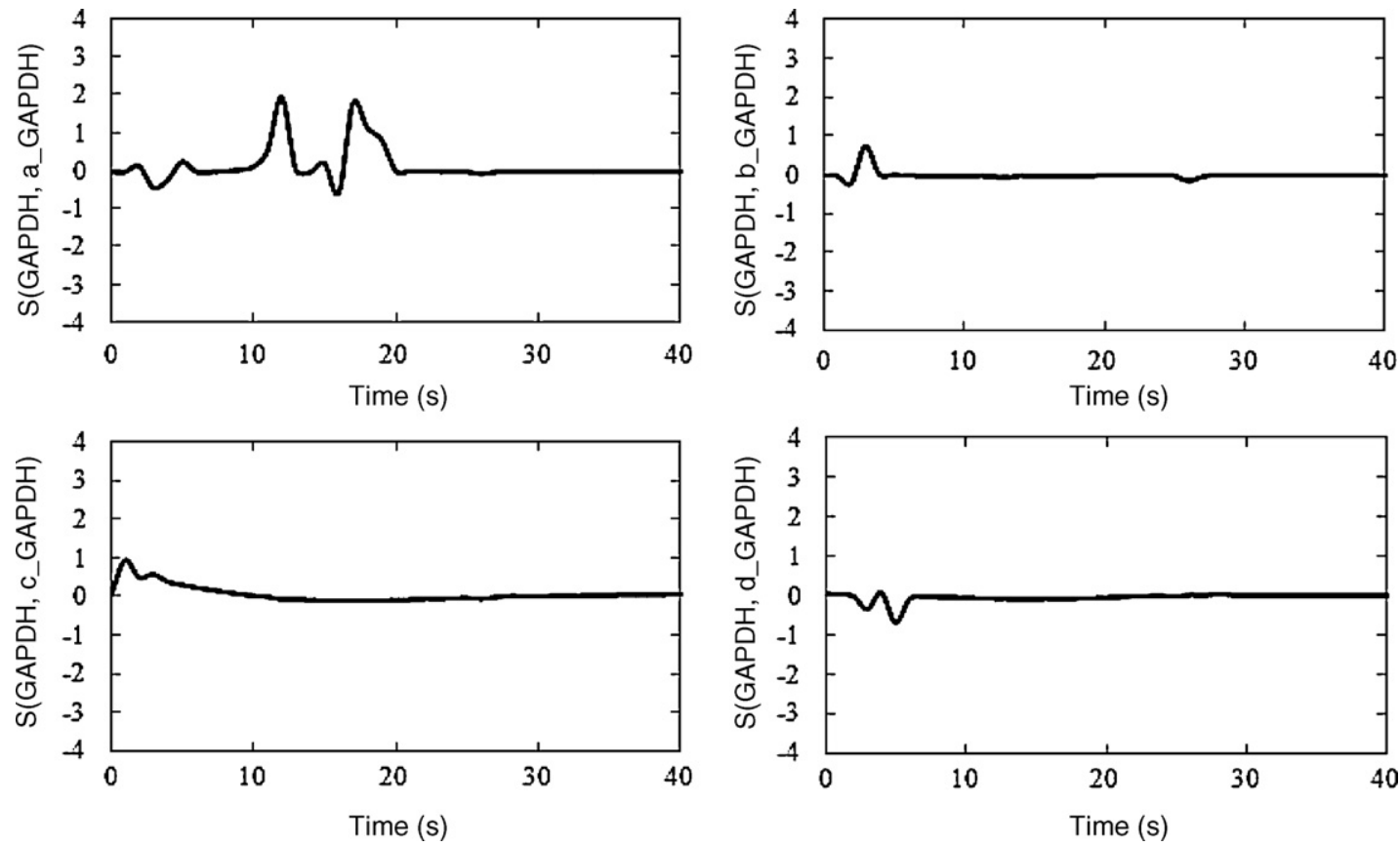

Fig. 4. Time-dependent sensitivity analysis of the kinetic parameters (a_GAPDH, b_GAPDH, c_GAPDH and d_GAPDH) on the flux through GAPDH reaction.

on time-dependent sensitivity analysis, according to Eq. (12). High parameter sensitivities are often indicative that some parts of the system have not been adequately described. Here, we performed as example this analysis on the flux through the GAPDH reaction, one of the main reactions of glycolysis, for the period of $40 \mathrm{~s}$. The time course results are presented in Fig. 4.

It is interesting to note the larger sensitivity with respect to a GAPDH parameter for the first $20 \mathrm{~s}$ and the relatively smaller effect of the other parameters. It is therefore interesting to focus on experiments on this period for fitting the kinetic parameters. Hence, with the sensitivity analysis we identified the most sensitive parameters and sampling time intervals, providing directions to future experimental design aimed at model refinement.

\section{Conclusions}

One of the major problems of setting-up large-scale dynamic models is the lack of kinetic data. The kinetic parameters are usually unknown, as well as the specific kinetic rate laws. Moreover, for a large number of reactions, the kinetic parameters are available in the literature only as general values obtained in in vitro conditions. There is therefore a need for alternative modeling approaches.

In this contribution we have build four alternative models combining Michaelis-Menten kinetics and approximated kinetic expressions, such as generalized mass action, convenience, lin-log, and power-law and the performance of these new models has been compared with a reference mechanistic model representing the $E$. coli central carbon metabolism.

Considering the good behavior performance obtained with the kinetic model composed of Michaelis-Menten and lin-log kinetics in comparison with the others, we conclude that this is a suitable approach for complex large-scale models where the exact rate laws are unknown. In combination with recent developments in time series data measurements, this approach should facilitate modeling of large-scale networks. As discussed above, care should be taken, however, if the conditions where the model is to be applied are far away from the reference state, as lin-log models are based on a local approximation assumption, and also when metabolite concentrations are close to zero.

\section{Acknowledgments}

Research supported by PhD grant SFRH/BD/25506/2005 from the Fundação para a Ciência e a Tecnologia (FCT) and the MITPortugal project "Bridging Systems and Synthetic Biology for the development of improved microbial cell factories" (MITPt/BS-BB/0082/2008). The authors thank Dr. C. Chassagnole, who provided the experimental data set.

\section{References}

Bakker, B.M., Michels, P.A.M., Opperdoes, F.R., Westerhoff, H.V., 1997. Glycolysis in bloodstream form Trypanosoma brucei can be understood in terms of the kinetics of the glycolytic enzymes. Journal of Biological Chemistry 272 (6), 3207-3215.

Bakker, B.M., Michels, P.A.M., Opperdoes, F.R., Westerhoff, H.V., 1999. What controls glycolysis in bloodstream form Trypanosoma brucei. Journal of Biological Chemistry 274 (21), 14551-14559.

Blattner, F.R., Plunkett, G.l., Perna, N.T., Burland, V., Riley, M., Collado-Vides, J., Glasner, J.D., Rode, C.K., Mayhew, G.F., Gregor, J., Davis, N.W., Kirpatrick, H.A., Goeden, M.A., Rose, D.J., Mau, B., Shao, Y., 1997. The complete genome sequence of E. coli K-12. Science 277 (5331), 1453-1462.

Buchholz, A., Hurlebaus, J., Wandrey, C., Takors, R., 2002. Metabolomics: quantification of intracellular metabolite dynamics. Biomolecular Engineering 19 (1), 5-15.

Buchholz, A., Takors, R., Wandrey, C., 2001. Quantification of intracellular metabolites in E. coli $\mathrm{K} 12$ using liquid chromatographic-electrospray ionization tandem mass spectrometric techniques. Analytical Biochemistry 295 (2), 129-137.

Chassagnole, C., Fell, D.A., Rais, B., Kudla, B., Mazat, J.P., 2001. Control of the threonine-synthesis pathway in Escherichia coli: a theoretical and experimental approach. Biochemical Journal 356 (2), 433-444.

Chassagnole, C., Noisommit-Rizzi, N., Schmid, J.W., Mauch, K., Reuss, M., 2002. Dynamic modeling of the central carbon metabolism of Escherichia coli. Biotechnology and Bioengineering 79 (1), 53-73.

Conrad, E.D., Tyson, J.J., 2006. Modelling molecular interaction networks with nonlinear ordinary differential equation. In: Szallasi, Z., Stelling, J., Periwal, V. (Eds.) Systems Modelling in Cellular Biology. MIT Press, Cambridge, pp. 97-123.

Cornish-Bowden, A., 1995. Fundamental of Enzyme Kinetics, 2nd edn. Portland Press, London.

Dano, S., Madsen, M.F., Schmidt, H., Cedersund, G., 2006. Reduction of a biochemical model with preservation of its basic dynamic properties. FEBS Journal 273 (21), 4862-4877.

Famili, I., Mahadevan, R., Palsson, B.O., 2005. k-Cone analysis: determining all candidate values for kinetic parameters on a network scale. Biophysical Journal 88 (3), 1616-1625.

Fogel, L.J., Owens, A.J., Walsh, M.J., 1966. Artificial Intelligence Through Simulated Evolution. Wiley, New York. 
Hatzimanikatis, V., Bailey, J.E., 1997. Effects of spatiotemporal variations on metabolic control: approximate analysis using (log)linear kinetic models. Biotechnology and Bioengineering 54 (2), 91-104.

Hatzimanikatis, V., Bailey, J.E., 1996. MCA has more to say. Journal of Theoretical Biology 182 (3), 233-242.

Heijnen, J.J., 2005. Approximative kinetic formats used in metabolic network modeling. Biotechnology and Bioengineering 91 (5), 534-545.

Heinrich, R., Schuster, S., 1996. The Regulation of Cellular Systems. Kluwer Academic Publisher.

Hooke, R., Jeeves, T.A., 1961. Direct search solution of numerical and statistical problems. Journal of the ACM (JACM) 8 (2), 212-229.

Hoops, S., Sahle, S., Gauges, R., Lee, C., Pahle, J., Simus, N., Singhhal, M., Xu, L., Mendes, P., Kummer, U., 2006. COPASI - a complex pathway simulator. Bioinformatics 22 (24), 3067-3074.

Horn, F., Jackson, R., 1972. General mass action kinetics. Archive for Rational Mechanics and Analysis 47 (2), 81-116.

Ishii, N., Suga, Y., Hagiya, A., Watanabe, H., Mori, H., Yoshino, M., Tomita, M., 2007. Dynamic simulation of an in vitro multi-enzyme system. FEBS Letters 581 (3), 413-420.

Jamshidi, N., Edwards, J.S., Fahland, T., Church, G.M., Palsson, B.O., 2001. Dynamic simulation of the human red blood cell metabolic network. Bioinformatics 17 (3), 286-287.

Johannes, K.J., Hess, B., 1973. Allosteric kinetics of pyruvate kinase of Saccharomyces carlsbergensis. Journal of Molecular Biology 76 (2), 181-205.

Ji, Z.L., Chen, X., Zhen, C.J., Yao, L.X., Han, L.Y., Yeo, W.K., Chung, P.C., Puy, H.S., Tay, Y.T., Muhammad, A., Chen, Y.Z., 2003. KDBI: kinetic data of bio-molecular interaction database. Nucleic Acids Research 31 (1), 255-257.

Karp, P., Riley, M., Saier, M., Paulsen, I.T., Paley, S.M., Pellegrini-Toole, A., 2000. The EcoCyc and MetaCyc databases. Nucleic Acids Research 28 (1), 56-59.

Kameshita, I., Tokushige, M., Izui, K., Katsuki, H., 1979. Phosphoenolpyruvate carboxylase of Escherichia coli affinity labeling with bromopyruvate. Journal of Biochemistry 86 (5), 1251-1257.

Kitano, H., 2002. Computational systems biology. Nature 420 (6912), 206-210.

Kitayama, T., Kinoshita, A., Sugimoto, M., Nakayama, Y., Tomita, M., 2006. A simplified method for power-law modelling of metabolic pathways from time-course data and steady-state flux profiles. Theoretical Biology and Medical Modelling 3, 24-33.

Klipp, E., Liebermeister, W., Helbig, A., Kowald, A., Schaber, J., 2007. Systems Biology standards - the community speaks. Nature Biotechnology 25 (4), 390-391.

Lauffenburger, D.A., Linderman, J.L., 1993. Receptors: Models for Binding, Trafficking, and Signaling. Oxford University Press, New York.

Le Novere, N., Bornstein, B., Broicher, A., Courtot, M., Donizelli, M., Dharuri, H., Li, L., Sauro, H., Schilstra, M., Shapiro, B., Hucka, M., 2006. BioModels database: free, centralized database of curated, published, quantitative kinetic models of biochemical and cellular systems. Nucleic Acids Research 34, D689-D691.

Liebermeister, W., Klipp, E., 2006. Bringing metabolic networks to life: convenience rate law and thermodynamic constraints. Theoretical Biology and Medical Modelling 3, 41-54.

Liao, J.C., Hou, S.Y., Chao, Y.P., 1996. Pathway analysis, engineering, and physiological considerations for redirecting central metabolism. Biotechnology and Bioengineering 52 (1), 129-140.

Mahadevan, R., Edwards, J.S., Doyle, F.J., 2002. Dynamic flux balance analysis of diauxic growth in Escherichia coli. Biophysical Journal 83 (3), 1331-1340.

Mendes, P., 2001. Modelling large biological systems from functional genomic data: parameter estimation. In: Kitano, H. (Ed.), Foundations in Systems Biology. MIT Press, Cambridge, MA, pp. 163-186.

Mendes, P., Kell, D.B., 1998. Non-linear optimization of biochemical pathways: applications to metabolic engineering and parameter estimation. Bioinformatics 14 (10), 869-883.

Moles, C.G., Mendes, P., Banga, J.R., 2003. Parameter estimation in biochemical pathways: a comparison of global optimization methods. Genome Research 13, 2467-2474.

Murray, J.D., 2002. Mathematical Biology. I. An Introduction, 3rd edn. SpringerVerlag, New York.

Nikerel, I.E., van Winden, W.A., van Gulik, W.M., Heijnen, J.J., 2006. A method for estimation of elasticities in metabolic networks using steady-state and dynamic metabolomics data and lin-log kinetics. BMC Bioinformatics 7, 540-563.

Petzold, L., 1983. Automatic selection of methods for solving stiff and nonstiff systems of ordinary differential equations. SIAM Journal of Scientific and Statistical Computing 4 (1), 136-148.

Preiss, J., Greenberg, E., Sabraw, A., 1975. Biosynthesis of bacterial glycogen. Kinetic studies of a glucose-1-phosphate adenylyltransferase (EC 2. 7. 7.27) from a glycogen-deficient mutant of Escherichia coli B. Journal of Biological Chemistry 250 (19), 7631-7638.

Ren, Q.C.K., Paulsen, I.T., 2007. TransportDB: a comprehensive database resource for cytoplasmic membrance transport systems and outer membrane channels. Nucleic Acids Research 35, D274-D279.

Richey, B., Cayley, D.S., Mossing, M.C., Kolka, C., Anderson, C.F., Farrar, T.C., Record, M.T., 1987. Variability of the intracellular Ionic environment of Escherichia coli -
Differences between in vitro and in vivo effects of ion concentrations on proteinDNA interactions and gene expression. Journal of Biological Chemistry 262 (15), 7157-7164.

Rodriguez-Fernandez, M., Mendes, P., Banga, J.R., 2006. A hybrid approach for efficient and robust parameter estimation in biochemical pathways. BioSystems 83 (2-3), 248-265

Rojas, I., Golebiewski, M., Kania, R., Krebs, O., Mir, S., Weidemann, A., Wittig, U., 2007. SABIO-RK: a database for biochemical reaction and their kinetics. BMC Systems Biology I, S6.

Savageau, M.A., 1969. Biochemical systems analysis. I. Some mathematical properties of rate law for component enzymatic reactions. Journal of Theoretical Biology 25 (3), 365-369.

Savageau, M.A., 1970. Biochemical systems analysis. 3. Dynamic solutions using a power-law approximation. Journal of Theoretical Biology 26 (2), 215-226.

Savageau, M.A., Voit, E.O., 1982. Power-law approach to model biochemical systems I. Fermentation Technology 60 (3), 221-228.

Schauer, M., Heinrich, R., 1979. Analysis of the quasi-steady-state approximation for an enzymatic one-substrate reaction. Journal of Theoretical Biology 79 (4), $425-442$.

Schauer, M., Heinrich, R., 1983. Quasi-steady-state approximation in the mathematical-modeling of biochemical reaction networks. Mathematical Biosciences 65 (2), 155-170.

Schomburg, I., Chang, A., Schomburg, D., 2002. BRENDA, enzyme data and metabolic information. Nucleic Acids Research 30 (1), 47-49.

Smallbone, K., Simeonidis, E., Broomhead, D.S., Kell, D.B., 2007. Something from nothing - bridging the gap between constraint-based and kinetic modelling. FEBS Journal 274 (21), 5576-5585.

Soga, T., Ohashi, Y., Ueno, Y., Naraoka, H., Tomita, M., Nishioka, T., 2003. Quantitative metabolome analysis using capillary electrophoresis mass spectrometry. Journal of Proteome Research 2 (5), 488-494.

Spieth, C., Hassis, N., Streichert, F., 2006. Comparing mathematical models on the problem of network inference. In: Proceedings of the Genetic and Evolutionary Computation Conference.

Sundararaj, S., Guo, A., Habibi-Nazhad, B., Rouani, M., Stothard, P., Ellison, M., Wishart, D.S., 2004. The CyberCell Database (CCDB): a comprehensive, selfupdating, relational database to coordinate and facilitate in silico modeling of Escherichia coli. Nucleic Acids Research 32, D293-D295.

Teusink, B., Passarge, J., Reijenga, C.A., Esgalhado, E., van der Weijden, C.C., Schepper, M. Walsh, M.C., Bakker, B.M., van Dam, K., Westerhoff, H.V., Snoep, J.L. 2000. Can yeast glycolysis be understood in terms of in vitro kinetics of the constituent enzymes? Testing biochemistry. European Journal of Biochemistry 267 (17), 5313-5329.

Theobald, U., Mailinger, W., Baltes, M., Rizzi, M., Reuss, M., 1997. In vivo analysis of metabolic dynamics in Saccharomyces cerevisae: I. Experimental observations. Biotechnology and Bioengineering 55 (2), 305-316.

Theobald, U., Mailinger, W., Reuss, M., Rizzi, M., 1993. In vivo analysis of glucoseinduced fast changes in yeast adenine-nucleotide pool applying a rapid sampling technique. Analytical Biochemistry 214 (1), 31-37.

Vaseghi, S., Baumeister, A., Rizzi, M., Reuss, M., 1999. In vivo dynamics of the pentose phosphate pathway in Saccharomyces cerevisae. Metabolic Engineering 1 (2), $128-140$.

Vilela, M., Chou, I.-C., Vinga, S., Vasconcelos, A.T.R., Voit, E., Almeida, S.J., 2008 Parameter estimation in S-systems models. BMC Systems Biology 2, 35-48.

Visser, D., Heijnen, J.J., 2002. The mathematics of metabolic control analysis revisited. Metabolic Engineering 4 (2), 114-123.

Visser, D., Heijnen, J.J., 2003. Dynamic simulation and metabolic re-design of a branched pathway using lin-log kinetics. Metabolic Engineering 5 (3), $164-176$.

Visser, D.v.Z.A., Heijnen, J.J., Frank, J., 2002. Rapid sampling for analysis of in vivo kinetics the BioScope: a system for continuous-pulse experiments. Biotechnology and Bioengineering 79 (6), 674-681.

Voit, E., Neves, A.R., Santos, H., 2006. The intricate side of systems biology. Proceedings of the National Academy of Sciences of the United States of America 103 (25), 9452-9457.

Wahl, S.A., Haunschild, M.D., Oldiges, M., Wiechert, W., 2006. Unravelling the regulatory structure of biochemical networks using stimulus response experiments and large-scale model selection. IEE Proceedings Systems Biology 153 (4), 275-285.

Wang, F.S., Ko, C.L., Voit, E.O., 2007. Kinetic modeling using S-systems and lin-log approaches. Biochemical Engineering Journal 33 (3), 238-247.

Wright, B.E., Butler, M., Albe, K.R., 1992. Systems analysis for the tricarboxylic acid cycle in Dictyostelium discoideum. The Journal of Biological Chemistry 267 (5), 3101-3105.

Yugi, K., Nakayama, Y., Kinoshita, A., Tomita, M., 2005. Hybrid dynamic/static method for large-scale simulation of metabolism. Theoretical Biology and Medical Modelling 2 (1), 42-53.

Zi, Z., Zheng, Y., Rundell, A.E., Klipp, E., 2008. SBML-SAT: a systems biology markup language (SBML) based sensitivity analysis tool. BMC Bioinformatics 9, 342-355. 